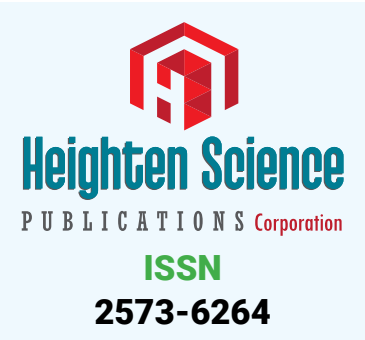

*Address for Correspondence: Jodie Adams, New Heights Physical Therapy, 5736 NE Glisan St, Portland Oregon, 97213, Portland, Email: Jodie@newheightstherapy.com

Submitted: 29 August 2017

Approved: 18 September 2017

Published: 19 September 2017

Copyright: @ 2017 Adams J, et al. This is an open access article distributed under the Creative Commons Attribution License, which permits unrestricted use, distribution, and reproduction in any medium, provided the original work is properly cited.
Case Report

\section{Frequency specific microcurrent resolves chronic pain and adhesions after ulnar transposition surgery}

\author{
Jodie Adams ${ }^{1 *}$ and Carolyn McMakin² \\ ${ }^{1}$ New Heights Physical Therapy, 5736 NE Glisan St, Portland Oregon, 97213, Portland \\ ${ }^{2}$ Fibromyalgia and myofascial pain clinic of Portland, Portland
}

\section{Summary}

Frequency specific microcurrent (FSM) has been used to treat myofascial pain syndrome $[1,2]$ fibromyalgia associated with spine trauma [3], delayed onset muscle soreness [4], acute and chronic neuropathic pain [5] and chronic scarring in burn patients [6]. In this case, its use was modified to include not only the treatment of neuropathic pain but the treatment of neural adhesions in a patient with a ten year history of pain at rest and painful restricted range of motion following an ulnar nerve anterior transposition surgery in 2001. Eleven standard physical therapy sessions including augmented soft tissue mobilization using plastic tools, and muscle strengthening exercises and stretching failed to resolve symptoms. Three sessions of frequency specific microcurrent produced complete resolution of pain and symptoms. Patient remained symptoms free at one-year follow up.

\section{Introduction}

Postoperative perineural scarring is a significant problem following ulnar nerve transposition surgery and the prognosis following surgery is worse when significant scarring occurs [7]. Dense scarring has been associated with poor outcomes when ulnar nerve transposition fails to alleviate symptoms. Re-operating on patients with a poor outcome from an initial transposition surgery produced fair to poor results in 55\% of patients. Only 4 of 9 patients experienced symptom resolution following neurolysis surgery [8].

Physical therapy has been discussed as an option in avoiding surgery in mild cases of ulnar compression neuropathy [9]. But there is no literature that discusses physical therapy options for treating an unsuccessful postsurgical outcome following ulnar nerve decompression surgery.

Neuropathic pain and adhesions following ulnar nerve transposition surgery are therefore typically difficult to treat within a medical and physical therapy treatment model. Manual physical therapists can perform neural mobilization techniques alone or in combination with joint mobilization to reduce pain and improve range of motion but the process can be slow, painful and difficult [10].

The purpose of treatment in this case was to discover if frequency specific microcurrent showed promise in the treatment of chronic neuropathic pain and adhesions following unsuccessful ulnar nerve anterior transposition surgery.

The patient was a 28-year-old male who presented on 8/22/2011 for treatment of hypersensitivity and severe left cubital and forearm pain. His pain had varied and persisted since an ulnar nerve transposition surgery in 2001. His first child was due to be born within two weeks of his initial treatment and he sought care so that he would be able to lift and carry his infant daughter. 
In recounting his history he stated that his left elbow pain began to gradually increase at age 13 following multiple falls and accidents that produced trauma to the left elbow such as skateboarding and bicycle accidents, an all-terrain-vehicle (ATV) accident and a fall from a one-story roof. By age 15, when he began driving, elbow flexion produced significant pain. Nerve conduction performed at age 19 demonstrated a reduction in conduction velocity across the ulnar nerve at the elbow and normal conduction across the wrist. The preoperative clinical assessment showed weakness in abductor digiti minimi, diminished sensation of the left fourth and fifth fingers and a very positive Tinnel's sign at the ulnar groove. The preoperative diagnosis was ulnar entrapment neuropathy.

No conservative therapy was attempted and ulnar nerve anterior transposition surgery was performed at age 19 in September 2001.

The operative report stated that the "ulnar nerve lay within the ulnar groove and appeared to be entrapped by fibrous bands adjacent to the flexor carpi ulnaris. There was no evidence of neuroma". "With operative magnification and microdissection, the nerve was mobilized and branches to the flexor carpi ulnaris spared. The mobilization allowed anterior transposition of the nerve. A notch was cut in the fascia above and below the transposition to avoid kinking of the nerve at the point of transposition. The ulnar grove was closed with non-absorbable \#3-0 silk suture. A subcutaneous pocket was fashioned for the nerve with \#3-0 Vicryl suture, with a final skin closure of \#3-0 Vicryl suture, \#5-0 Monocryl and Steri-Strips."

Surgery produced no significant change in pain and the patient had no follow up therapy. He experienced increased cutaneous sensitivity and pain in the area over the following 10 years.

In 2010 at age 28, he initiated physical therapy for "left elbow, forearm and hand pain that had worsened over time" since the surgery. His VAS pain score was $5 / 10$. Between February 2 and April 12, 2010 he received eleven physical therapy treatments. He was treated nine times with assisted soft tissue therapy using acrylic tools (ASTYM) with the intention of breaking up soft tissue adhesions. He was treated with e-stim and ice on four of those eleven visits. He had exercise therapy at every visit that included mobilization of the median and ulnar nerves, foam roller thoracic mobilization, proneball scapular strengthening and biceps, triceps and latissimus dorsi strengthening with hand weights. His pre-treatment TAOS was $86 \%$ and his post-treatment was $92 \%$ at the time of discharge. His VAS pain score at discharge was $4 / 10$.

He sought additional physical therapy at our facility in August 2011 due to increased pain and concern that he would not be able to hold his infant daughter. He stated that prior physical therapy had produced temporary reduction in pain but did not change the hyperesthesia or tingling. Elbow pain had gradually increased over the intervening year and was rated at a $7 / 10$ on a VAS pain scale at this evaluation. The Care Connection, TAOS functional index score was $80 \%$ with sleeping, recreation, carrying and driving being most restricted at $3 / 5$ and personal care and lifting restricted at 4/5.

The initial evaluation showed elbow range of motion to be full but painful past 95 degrees of flexion in the elbow. Elbow extension was his position of comfort. Shoulder abduction and flexion caused pain in the left forearm and elbow. Shoulder flexion was 127 degrees with scapular elevation and shoulder abduction was 132 degrees. Wrist range of motion was normal but painful with repetition.

Manual muscle testing of elbow flexion/extension was 4/5 with pain, wrist supination/pronation was $4 / 5$ with pain. Upper limb tension testing was positive with pain in the median and ulnar nerve distributions. Sensory testing for light touch showed hyperesthesia and pain in the ulnar nerve cutaneous distributions in the medial arm and forearm. He could not tolerate any touch or pressure in these areas and couldn't rest his arm on any surface. 


\section{Materials and Methods}

The first treatment in August 2011 included Frequency Specific Microcurrent (FSM) to reduce nerve pain so the patient would tolerate manual therapy and gentle soft tissue mobilization. The patient tolerated treatment well and had some temporary pain reduction. He returned one month later for a second treatment in September 2011. At this treatment the frequencies described as "reducing scar tissue in the nerve" [11] were applied with the limb at rest and while performing passive nerve glide techniques within a pain free range.

\section{Photograph 1: Contact placement for elbow treatment}

Caption: Treatment contacts are set up with the positive leads in a warm wet towel wrapped around the neck where the nerve exits the spine and the negative leads wrapped in a warm wet towel at the end of the nerve to be treated, near the elbow. The therapist uses the hands under the contacts to mobilize the forearm nerve and fascia.

Frequency Specific Microcurrent uses a frequency thought to address a certain pathology, such as inflammation or scarring on one channel and a frequency describing a certain tissue on the second channel. The frequency combination observed to reduce nerve pain is 40 hertz on channel $\mathrm{A}$ and $396 \mathrm{~Hz}$ on channel B [11]. The frequency observed to increase range of motion and reduce scar tissue density in the nerve was 13 hertz on channel A and 396 hertz on channel B. 396 hertz is thought to be the frequency that affects the nerve as a tissue. 40 hertz is the frequency that has been observed to reduce inflammation and pain [3]. 13 hertz is thought to be the frequency that softens or dissolves scar tissue [6].

Frequency Specific Microcurrent was developed in 1996 when frequencies from a list created with a device manufactured in 1922 were applied with a two-channel microcurrent device instead of the original electrical equipment. Microcurrent devices, while approved in the category of TENS (transcutaneous electrical nerve stimulation) do not function as TENS devices since the subsensory current is 1000 times less than TENS. The original equipment has never been available for examination and the list was used as if the verbal descriptions of the frequencies were correct [12].

At the second treatment performed in September 2011, a two-channel Precision Micro (Precision Microcurrent, Newberg Oregon) was used to apply 40 hertz on channel A and 396 hertz on channel B for 30 minutes. Two leads from each channel were inserted into two graphite gloves that were wrapped in warm wet hand towels to provide broader current distribution and good conductivity. The polarized pulsed positive direct (DC) current was applied with the positive leads at the neck and the negative leads at the left hand. The current levels were set at 200 microamps and a medium wave slope was used. This application has been observed to reduce nerve pain and hyperesthesia [5].

The patient's pain and palpatory sensitivity were eliminated after 30 minutes treatment using 40 hertz and 396 hertz. Range of motion was still restricted due to pain. The device settings were then changed to deliver 13 hertz on channel A and 396 hertz on channel B [11]. Gentle passive range of motion within the pain free range at the elbow and wrist was performed for 30 minutes. During this movement phase of treatment, 40 hertz on channel A and 396 hertz on channel B were used several times for 5 minutes to eliminate increases in pain caused by movement. Once pain was reduced the frequencies were returned to 13 hertz and 396 hertz and passive movement was resumed. It was observed that 13 hertz had no effect on pain and 40 hertz had no effect to increase range. In the last 15 minutes of treatment the patient performed active range of motion in the elbow, wrist and shoulder with no increase in pain. At the end of treatment, range of motion was full and pain free and cutaneous hyperesthesia had been eliminated (Table 1). 


\begin{tabular}{|c|c|}
\hline 2010 Pre Treatment 2010 & Post treatment-Standard PT-11 Sessions \\
\hline TAOS* $86 \%$ & TAOS* $92 \%$ \\
\hline VAS* $5 / 10$ & VAS $4 / 10$ \\
\hline 2011 Pre FSM Treatment & 2011 Post FSM Treatment -3 Sessions \\
\hline TAOS* $80 \%$ & Full function self report, patient self discharge \\
\hline VAS $7 / 10$ & VAS $0 / 10$ \\
\hline \multicolumn{2}{|c|}{2012 Post FSM treatment-Follow-up } \\
\hline \multicolumn{2}{|l|}{ TAOS $100 \%$} \\
\hline \multicolumn{2}{|l|}{ VAS $0 / 10$} \\
\hline *TAOS functional index score. & \\
\hline
\end{tabular}

\section{Results and Discussion}

At follow-up one week later the patient was pain free with full range of motion but complained of hyperesthesia at the surgical scar site. The device was applied as previously described; 40 hertz was used on one channel and 396 hertz was used on the second channel until the hyperesthesia resolved. The patient self-discharged after this treatment.

At follow-up one year later, in 2012, the patient rated his pain as $0 / 10$ on a VAS scale. TAOS functional index was $100 \%$ improved from $80 \%$. He rated himself as $7 / 7$ perceived improvement on Global Rating of Change scale. Physical examination showed full pain free range of motion at the shoulder, elbow and wrist. Manual muscle testing of the wrist and elbow was 5/5. He had no pain on palpation in the arm or forearm. Sensation was diminished in the medial arm and forearm but not hypersensitive as it was prior to treatment. He still reported pain when resting his elbow on a hard surface but stated that it did not impair any activities. He reported being able to hold his infant daughter comfortably with his left arm from the time of her birth through the one-year follow up. The patient reports that he remains pain free with full range of motion in 2017.

\section{Conclusion}

Frequency Specific Microcurrent shows promise in the treatment of neuropathic pain and neural adhesions in failed ulnar nerve transposition surgery and provided complete relief of symptoms after three treatment sessions when eleven sessions of standard physical therapy had failed. It is possible that this treatment may also be useful as conservative treatment to be used instead of or prior to surgery. Further research should be conducted.

\section{References}

1. McMakin C. Microcurrent Treatment of Myofascial Pain in the Head, Neck and Face. Top Clin Chiro. 1998; 5: 29-35. Ref.: https://goo.gl/pBnPm5

2. McMakin C. Microcurrent therapy: a novel treatment method for chronic low back myofascial pain. J Bodyw Mov Ther. 2004; 8: 143-153. Ref.: https://goo.gl/yxbgvM

3. McMakin C, Gregory W, Phillips T. Cytokine changes with microcurrent therapy of fibromyalgia associated with cervical trauma. J Bodyw Mov Ther. 2005; 9: 169-176. Ref.: https://goo.gl/jNLRPc

4. Curtis $D$, Fallows $S$, Morris M, McMakin C. The efficacy of frequency specific microcurrent therapy on delayed onset muscle soreness. J Bodyw Mov Ther. 2010; 14: 272-279. Ref.: https://goo.gl/Dt6o2q

5. McMakin C. Non-pharmacologic treatment of neuropathic pain using Frequency Specific Microcurrent. The Pain Practitioner. 2010; 68-73.

6. Huckfeldt R, Mikkelson K, Larson L. The Use of Microcurrent and Autocatalytic Silver Plated Nylon Dressings in Human Burn Patients: A Feasibility study. Maui, Hawaii: Proceedings of John Boswick Burn and Wound Symposium. 2003.

7. Leffert, RD. Anterior submuscular transposition of the ulnar nerves by the Learmonth technique. $J$ Hand Surg Am. 1982; 7: 147-155. Ref.: https://goo.gl/8YW4MM 
8. Dagregorio G, Saint-Cast $\mathrm{Y}$. Simple neurolysis for failed anterior submuscular transposition of the ulnar nerve at the elbow. Int Orthop. 2004; 28: 342-346. Ref.: https://goo.gl/rZkqCi

9. Robertson C, Saratsiotis J. A review of compressive ulnar neuropathy at the elbow. J Manipulative Physio Ther. 2005; 28: 345. Ref.: https://goo.gl/U9ae9z

10. Butler DS. Mobilization of the nervous system. Churchill Livingston. 1991.

11. McMakin C. Treating neuropathic pain, Elsevier Science Press, Edinburgh, Frequency Specific Microcurrent in Pain Management. Textbook for practitioners. 2010; 41-72.

12. McMakin C. Resurrecting Resonance, North Atlantic Books, Berkeley, CA. The Resonance Effect. 2017; 10-14. 